\title{
ON A RELATIONSHIP BETWEEN NORMAL-SHEAR COEFFICIENTS AND TENSILE MODULI IN ANISOTROPIC MATERIALS
}

\begin{abstract}
Summary
In general, stress and deformation tensors of anisotropic linear elastic materials are related by the fourth order compliance/elasticity tensor. It has been observed that in the case of a planar (2D) orthotropic material the off-diagonal component of the compliance tensor known as normal-shear coefficient is equal to one half of the derivative of corresponding tensile compliance with respect to the angle of rotation of coordinate axes. A detailed analysis of the transformation law for the components of the compliance tensor is given, including the proof that the same relation also holds in the general case of planar anisotropic materials. In the selected illustrative examples polar diagrams of tensile moduli and normal-shear coefficients are shown for representative cases of woven fabrics and textile composites.
\end{abstract}

Key words: anisotropy, elastic material properties, transformation of tensor components

\section{Introduction}

In the description of deformational behaviour of textile materials, such as woven or knitted fabrics and composites, the general form of Hooke's law for anisotropic planar materials can be applied for the cases of in-plane load and deformation, at least in a limited range where generally nonlinear response can be linearized. It appears that the existence of unexpected non-zero normal-shear coefficients can be detected as a cause of unwanted "skewing" (loss of orthogonality between warp and weft yarns after weaving) in some types of woven fabrics, resulting in problems in textile production and processing [1]. For that reason, the normal-shear coefficients as components of the compliance tensor deserve careful attention. While they are equal to zero in principal material directions, they take non-zero values in the general rotated directions of coordinate system [2, 3].

A systematic approach to elastic properties of orthotropic materials, with an emphasis on tensor invariants, is given in [4] by Alfirević and Skozrit. In [5] it has been observed as an interesting fact that the double normal-shear coefficient coincides with the rate of change of the corresponding tensile compliance (inverse of tensile modulus) with respect to the angle of rotation of coordinate axes. The content of section 2 of this paper shows that this is not accidental, but rather follows from the general rules of tensor components transformation. To the author's knowledge this simple relation has not been mentioned in the literature, possibly 
because expressions for general components of the fourth order elasticity/compliance tensors take a somewhat complex form.

In the deformational characterization of $2 \mathrm{D}$ anisotropic materials it is usually convenient to represent tensile properties (elastic moduli) using a polar diagram. This gives grounds for equally convenient representation of normal-shear coefficients based on the geometric meaning of the first derivative. In section 3, some examples are provided in which this is illustrated on a selection of cases representative for woven fabrics and textile composites.

\section{Elastic material properties in initial and rotated coordinate systems}

Hooke's law for an isotropic material under plane stress can be written in the vector matrix notation in the following form:

$$
\left\{\begin{array}{c}
\varepsilon_{x} \\
\varepsilon_{y} \\
\gamma_{x y}
\end{array}\right\}=\left[\begin{array}{ccc}
1 / E & -v / E & 0 \\
-v / E & 1 / E & 0 \\
0 & 0 & 1 / G
\end{array}\right]\left\{\begin{array}{l}
\sigma_{x} \\
\sigma_{y} \\
\tau_{x y}
\end{array}\right\}=\frac{1}{E}\left[\begin{array}{ccc}
1 & -v & 0 \\
-v & 1 & 0 \\
0 & 0 & 2(1+v)
\end{array}\right]\left\{\begin{array}{l}
\sigma_{x} \\
\sigma_{y} \\
\tau_{x y}
\end{array}\right\}
$$

In the case of an orthotropic material Hooke's law takes a quite similar form for principal material directions; the differences from (1) are that principal tensile moduli (Young's moduli) can generally be different and shear modulus $\mathrm{G}$ can be independent:

$$
\left\{\begin{array}{c}
\varepsilon_{T} \\
\varepsilon_{L} \\
\gamma_{T L}
\end{array}\right\}=\left[\begin{array}{ccc}
1 / E_{T} & -v_{L T} / E_{T} & 0 \\
-v_{T L} / E_{L} & 1 / E_{L} & 0 \\
0 & 0 & 1 / G_{T L}
\end{array}\right]\left\{\begin{array}{c}
\sigma_{T} \\
\sigma_{L} \\
\tau_{T L}
\end{array}\right\}
$$

Indices $\mathrm{T}$ and $\mathrm{L}$ have the meaning of "transversal" and "longitudinal" as principal material directions. Poisson's ratios $v_{\mathrm{LT}}$ and $v_{\mathrm{TL}}$ are related by the condition of symmetry of the compliance matrix in (2).

In the case of general rotated coordinate axes $\mathrm{x}, \mathrm{y}$ the above relation takes the general form in which the off-diagonal zero components of the matrix in (2) are replaced by the normal-shear coefficients $\alpha$ :

$$
\left\{\begin{array}{c}
\varepsilon_{x} \\
\varepsilon_{y} \\
\gamma_{x y}
\end{array}\right\}=\left[\begin{array}{ccc}
1 / E_{x} & -v_{y x} / E_{x} & \alpha_{x} \\
-v_{x y} / E_{y} & 1 / E_{y} & \alpha_{y} \\
\alpha_{x} & \alpha_{y} & 1 / G_{x y}
\end{array}\right]\left\{\begin{array}{c}
\sigma_{x} \\
\sigma_{y} \\
\tau_{x y}
\end{array}\right\}
$$

Elastic properties, i.e. components of the compliance matrix in (3) can be expressed in terms of principal material properties and the angle of rotation of the coordinate axes between $\mathrm{T}, \mathrm{L}$ and $\mathrm{x}, \mathrm{y}$. To this end, it is sufficient to apply the transformation law for the second order stress and strain tensors, see Fig. 1.

a)
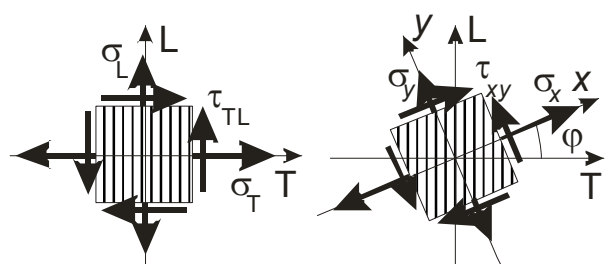

b)

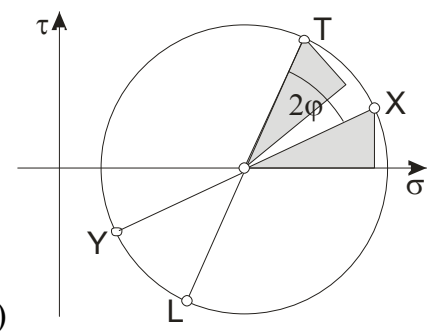

Fig. 1 Stress components in orthotropic material in principal and general directions (a), Mohr's circle (b) 
Normal-Shear Coefficients and

Tensile Moduli in Anisotropic Materials

According to the graphical representation in Mohr's circle, Fig. 1.b, the stress components in the $\mathrm{T}, \mathrm{L}$ directions can be expressed in terms of components in $\mathrm{x}, \mathrm{y}$ directions as

$$
\begin{aligned}
& \sigma_{T}=\frac{\sigma_{x}+\sigma_{y}}{2}+\frac{\sigma_{x}-\sigma_{y}}{2} \cos 2 \varphi-\tau_{x y} \sin 2 \varphi=\sigma_{x} \cos ^{2} \varphi+\sigma_{y} \sin ^{2} \varphi-2 \tau_{x y} \sin \varphi \cos \varphi \\
& \sigma_{L}=\frac{\sigma_{x}+\sigma_{y}}{2}-\frac{\sigma_{x}-\sigma_{y}}{2} \cos 2 \varphi+\tau_{x y} \sin 2 \varphi=\sigma_{x} \sin ^{2} \varphi+\sigma_{y} \cos ^{2} \varphi+2 \tau_{x y} \sin \varphi \cos \varphi \\
& \tau_{T L}=\frac{\sigma_{x}-\sigma_{y}}{2} \sin 2 \varphi+\tau_{x y} \cos 2 \varphi=\sigma_{x} \sin \varphi \cos \varphi-\sigma_{y} \sin \varphi \cos \varphi+\tau_{x y}\left(\cos ^{2} \varphi-\sin ^{2} \varphi\right)
\end{aligned}
$$

A similar inverse transformation holds for the rotation of the coordinate axes from $\mathrm{T}, \mathrm{L}$ to $\mathrm{x}, \mathrm{y}$ and written for the strain components it reads

$$
\begin{aligned}
& \varepsilon_{x}=\varepsilon_{T} \cos ^{2} \varphi+\varepsilon_{L} \sin ^{2} \varphi+\gamma_{T L} \sin \varphi \cos \varphi \\
& \varepsilon_{y}=\varepsilon_{T} \sin ^{2} \varphi+\varepsilon_{L} \cos ^{2} \varphi-\gamma_{T L} \sin \varphi \cos \varphi \\
& \gamma_{x y}=-2 \varepsilon_{T} \sin \varphi \cos \varphi+2 \varepsilon_{L} \sin \varphi \cos \varphi+\gamma_{T L}\left(\cos ^{2} \varphi-\sin ^{2} \varphi\right)
\end{aligned}
$$

Now substitute (4) in (2) and then the obtained expressions for $\varepsilon_{\mathrm{T}}, \varepsilon_{\mathrm{L}}$ and $\gamma_{\mathrm{TL}}$ introduce into (5). The comparison of the results with (3) reveals that for the elastic properties in the $\mathrm{x}$, y directions it holds:

$$
\begin{aligned}
& \frac{1}{E_{x}}=\frac{1}{E_{T}} \cos ^{4} \varphi+\frac{1}{E_{L}} \sin ^{4} \varphi+\left(\frac{1}{G_{T L}}-2 \frac{v_{T L}}{E_{L}}\right) \cos ^{2} \varphi \sin ^{2} \varphi \\
& \frac{1}{E_{y}}=\frac{1}{E_{T}} \sin ^{4} \varphi+\frac{1}{E_{L}} \cos ^{4} \varphi+\left(\frac{1}{G_{T L}}-2 \frac{v_{T L}}{E_{L}}\right) \cos ^{2} \varphi \sin ^{2} \varphi \\
& \frac{1}{G_{x y}}=\frac{1}{G_{T L}}\left(\cos ^{2} \varphi-\sin ^{2} \varphi\right)^{2}+4\left(\frac{1}{E_{T}}+\frac{1}{E_{L}}+2 \frac{v_{T L}}{E_{L}}\right) \cos ^{2} \varphi \sin ^{2} \varphi \\
& \frac{v_{x y}}{E_{y}}=\frac{v_{T L}}{E_{L}}\left(\cos ^{4} \varphi+\sin ^{4} \varphi\right)+\left(\frac{1}{G_{T L}}-\frac{1}{E_{T}}-\frac{1}{E_{L}}\right) \cos ^{2} \varphi \sin ^{2} \varphi \\
& \alpha_{x}=2 \sin \varphi \cos \varphi\left[\frac{1}{E_{L}} \sin ^{2} \varphi-\frac{1}{E_{T}} \cos ^{2} \varphi+\left(\frac{1}{2 G_{T L}}-\frac{v_{T L}}{E_{L}}\right)\left(\cos ^{2} \varphi-\sin ^{2} \varphi\right)\right] \\
& \alpha_{y}=2 \sin \varphi \cos \varphi\left[\frac{1}{E_{L}} \cos ^{2} \varphi-\frac{1}{E_{T}} \sin ^{2} \varphi-\left(\frac{1}{2 G_{T L}}-\frac{v_{T L}}{E_{L}}\right)\left(\cos ^{2} \varphi-\sin ^{2} \varphi\right)\right]
\end{aligned}
$$

The above expressions (6) represent the transformation rules for the $2 \mathrm{D}$ fourth order tensor in the case of orthotropic material compliance. They can be found in [2] and [3] in a somewhat rearranged form, and with seemingly a typing error in the expression for $\alpha_{x}$.

It is known in the analysis of plane stress that the derivative of the diagonal components in the matrix of the stress tensor is proportional to the off-diagonal component. For example, consider the expressions (4) and (5) to find that it holds

$$
2 \tau_{T L}=-\frac{d \sigma_{T}}{d \varphi} \quad, \quad \gamma_{x y}=\frac{d \varepsilon_{x}}{d \varphi}
$$


A close inspection of expressions (6) however reveals that there is also a very similar relation between tensile compliance $1 / \mathrm{E}$ and normal-shear coefficient $\alpha$ :

$$
\frac{d\left(\frac{1}{E_{x}}\right)}{d \varphi}=2 \alpha_{x}
$$

This property of compliance tensor seems to have remained unnoticed, since, at least to the author's knowledge, there is no mention of it in the literature.

The relation (8) was reported in [5], and the question arises if it might be valid not only for orthotropic but generally for anisotropic materials. Let us prove in the following consideration that this is true.

Start by reviewing the basic transformation rule for vector components in the rotated coordinate system, Fig. 2.

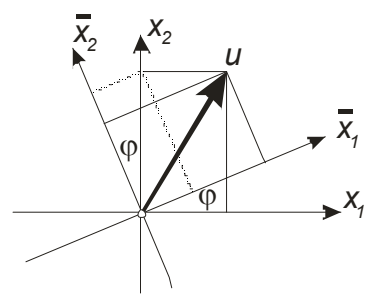

Fig. 2 Vector components in initial and rotated coordinate systems

The figure shows that the components in the rotated coordinate system can be expressed in terms of the components in the initial coordinate system as

$$
\bar{u}_{1}=u_{1} \cos \varphi+u_{2} \sin \varphi, \quad \bar{u}_{2}=-u_{1} \sin \varphi+u_{2} \cos \varphi
$$

In the index notation, introducing direction cosines, this rule takes the following form

$$
\bar{u}_{i}=a_{i j} u_{j}, \quad a_{i j}=\cos \left(\bar{x}_{i}, x_{j}\right), \quad i, j=1,2
$$

Taking the derivative of (9) with respect to the angle of the rotation of the coordinate system, the rates of change of the components in the rotated coordinate system are

$$
\frac{d \bar{u}_{1}}{d \varphi}=-u_{1} \sin \varphi+u_{2} \cos \varphi=\bar{u}_{2}, \quad \frac{d \bar{u}_{2}}{d \varphi}=-u_{1} \cos \varphi-u_{2} \sin \varphi=-\bar{u}_{1}
$$

Now, let two vectors be related by a symmetric second order tensor. For example, in the case of stress tensor these two vectors are the internal force on a section and the normal vector of the section area, while in the case of small strain these vectors are relative displacement and corresponding diagonal in a rectangular element. If the vectors and the tensor are denoted by $\boldsymbol{u}, \boldsymbol{v}$ and $T$ respectively, the relation reads

$$
u_{i}=T_{i j} v_{j} \quad ; \quad u_{1}=T_{11} v_{1}+T_{12} v_{2}, \quad u_{2}=T_{21} v_{1}+T_{22} v_{2}
$$

When the coordinate system rotates, the components of both vectors and the tensor $T$ depend on the angle $\varphi$ and taking the derivative of (12) we have 


$$
\begin{aligned}
& \frac{d u_{1}}{d \varphi}=\frac{d T_{11}}{d \varphi} v_{1}+T_{11} \frac{d v_{1}}{d \varphi}+\frac{d T_{12}}{d \varphi} v_{2}+T_{12} \frac{d v_{2}}{d \varphi} \\
& \frac{d u_{2}}{d \varphi}=\frac{d T_{21}}{d \varphi} v_{1}+T_{21} \frac{d v_{1}}{d \varphi}+\frac{d T_{22}}{d \varphi} v_{2}+T_{22} \frac{d v_{2}}{d \varphi}
\end{aligned}
$$

Substituting the derivatives of both vector components according to (11) and using (12) equations (13) become

$$
\begin{aligned}
& T_{21} v_{1}+T_{22} v_{2}=\frac{d T_{11}}{d \varphi} v_{1}+T_{11} v_{2}+\frac{d T_{12}}{d \varphi} v_{2}-T_{12} v_{1} \\
& -T_{11} v_{1}-T_{12} v_{2}=\frac{d T_{21}}{d \varphi} v_{1}+T_{21} v_{2}+\frac{d T_{22}}{d \varphi} v_{2}-T_{22} v_{1}
\end{aligned}
$$

These equations can be rewritten in the following form:

$$
\begin{aligned}
& \left(T_{21}+T_{12}-\frac{d T_{11}}{d \varphi}\right) v_{1}+\left(T_{22}-T_{11}-\frac{d T_{12}}{d \varphi}\right) v_{2}=0 \\
& \left(-T_{11}+T_{22}-\frac{d T_{21}}{d \varphi}\right) v_{1}-\left(T_{12}+T_{21}+\frac{d T_{22}}{d \varphi}\right) v_{2}=0
\end{aligned}
$$

Equations (15) hold for the arbitrary vector $v$, therefore the expressions in parentheses must vanish. Considering also the symmetry of the tensor $T$ it means that

$$
\frac{d T_{11}}{d \varphi}=2 T_{12}, \quad \frac{d T_{12}}{d \varphi}=T_{22}-T_{11}, \quad \frac{d T_{22}}{d \varphi}=-2 T_{12}
$$

This is in line with (7) and with the notion of the first invariant $T_{11}+T_{22}=$ const.

In the case of stress tensor, relations (16) can clearly be obtained by taking the derivative of the transformation rule (4). Still, it may be instructive and interesting to derive them directly from the conditions of equilibrium for an element in which one edge is slightly rotated by the angle $d \varphi$, see the right-hand side of Fig. 3.
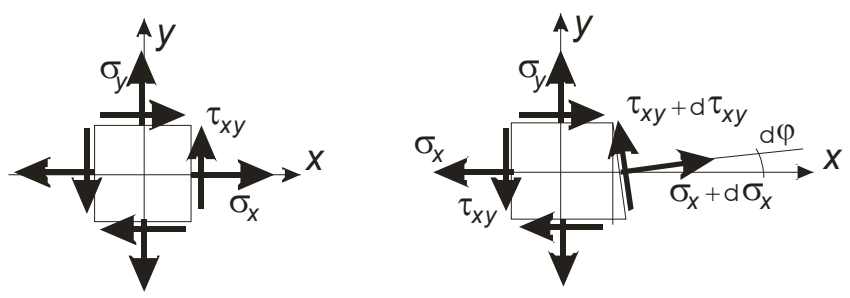

Fig. 3 Square element under plane stress and rotation of one edge by $d \varphi$

$$
\begin{aligned}
& \sum F_{x}=0, \quad\left(\sigma_{x}+d \sigma_{x}\right) t \frac{a}{\cos d \varphi} \cos d \varphi-\sigma_{x} a t+\tau_{x y} a t-\tau_{x y} a(1+\tan d \varphi) t- \\
& -\left(\tau_{x y}+d \tau_{x y}\right) \frac{a}{\cos d \varphi} t \sin d \varphi=0 \\
& \sum F_{y}=0, \quad \sigma_{y} a t-\sigma_{y} a(1+\tan d \varphi) t+\left(\sigma_{x}+d \sigma_{x}\right) \frac{a}{\cos d \varphi} t \sin d \varphi+ \\
& +\left(\tau_{x y}+d \tau_{x y}\right) \frac{a}{\cos \varphi} t \cos \varphi-\tau_{x y} a t=0
\end{aligned}
$$


In the equilibrium conditions (17) $t$ and $a$ denote the thickness and the side length of the square element respectively. For the small angle $d \varphi, \tan d \varphi$ can be raplaced by $d \varphi$ and after neglecting the small quantities of higher order equations (17) are reduced to

$$
\frac{d \sigma_{x}}{d \varphi}=2 \tau_{x y}, \quad \frac{d \tau_{x y}}{d \varphi}=\sigma_{y}-\sigma_{x}
$$

This is obviously in accordance with (16) and identical to the results of taking derivatives of the transformation rule for the stress components.

Now, consider the case of two second order tensors being related by a fourth order tensor. Let these tensors be second order tensors of stress and strain and a fourth order tensor of the elastic material compliance:

$$
\varepsilon_{i j}=S_{i j k l} \sigma_{k l}, \quad\left\{\begin{array}{l}
\varepsilon_{11} \\
\varepsilon_{22} \\
\varepsilon_{12} \\
\varepsilon_{21}
\end{array}\right\}=\left[\begin{array}{llll}
S_{1111} & S_{1122} & S_{1112} & S_{1121} \\
S_{2211} & S_{2222} & S_{2212} & S_{2221} \\
S_{1211} & S_{1222} & S_{1212} & S_{1221} \\
S_{2111} & S_{2122} & S_{2112} & S_{2121}
\end{array}\right]\left\{\begin{array}{l}
\sigma_{11} \\
\sigma_{22} \\
\sigma_{12} \\
\sigma_{21}
\end{array}\right\}
$$

The ordering in the vector - matrix form of (19) is done for ease of comparison with (3), from which it follows:

$$
\begin{aligned}
& \sigma_{11}=\sigma_{x}, \quad \sigma_{22}=\sigma_{y}, \quad \sigma_{12}=\sigma_{21}=\tau_{x y} \\
& \varepsilon_{11}=\varepsilon_{x}, \quad \varepsilon_{22}=\varepsilon_{y}, \quad \varepsilon_{12}=\varepsilon_{21}=\frac{1}{2} \gamma_{x y} \\
& S_{1111}=\frac{1}{E_{x}}, \quad S_{2222}=\frac{1}{E_{y}}, \quad S_{1122}=-\frac{v_{y x}}{E_{x}}, \quad S_{1112}=S_{1121}=\frac{1}{2} \alpha_{x} \\
& S_{2212}=S_{2221}=\frac{1}{2} \alpha_{y}, \quad S_{1212}=S_{1221}=S_{2112}=S_{2121}=\frac{1}{4 G_{x y}}
\end{aligned}
$$

Note the multiple symmetry in the matrix in (19), see also [3] page 309. The first component equation of (19) takes the following form:

$$
\varepsilon_{11}=S_{1111} \sigma_{11}+S_{1122} \sigma_{22}+2 S_{1112} \sigma_{12}
$$

Take the first derivative of (21) to obtain:

$$
\begin{aligned}
& \frac{d \varepsilon_{11}}{d \varphi}=\frac{d S_{1111}}{d \varphi} \sigma_{11}+S_{1111} \frac{d \sigma_{11}}{d \varphi}+\frac{d S_{1122}}{d \varphi} \sigma_{22}+S_{1122} \frac{d \sigma_{22}}{d \varphi}+ \\
& +2 \frac{d S_{1112}}{d \varphi} \sigma_{12}+2 S_{1112} \frac{d \sigma_{12}}{d \varphi}
\end{aligned}
$$

Taking the properties of derivatives of the second order tensor components (16) into account, equation (22) becomes:

$$
\begin{aligned}
& 2 \varepsilon_{12}=\frac{d S_{1111}}{d \varphi} \sigma_{11}+2 S_{1111} \sigma_{12}+\frac{d S_{1122}}{d \varphi} \sigma_{22}-2 S_{1122} \sigma_{12}+ \\
& +2 \frac{d S_{1112}}{d \varphi} \sigma_{12}+2 S_{1112}\left(\sigma_{22}-\sigma_{11}\right)
\end{aligned}
$$


Normal-Shear Coefficients and

Tensile Moduli in Anisotropic Materials

Once the left-hand side of (23) is replaced by the appropriate expression according to (19) (third or fourth component equation of (19)), the last equation (23) takes the following form:

$$
\begin{aligned}
& 2\left(S_{1211} \sigma_{11}+S_{1222} \sigma_{22}+2 S_{1212} \sigma_{12}\right)=\sigma_{11}\left(\frac{d S_{1111}}{d \varphi}-2 S_{1112}\right)+ \\
& +\sigma_{22}\left(\frac{d S_{1122}}{d \varphi}+2 S_{1112}\right)+2 \sigma_{12}\left(S_{1111}-S_{1122}+\frac{d S_{1112}}{d \varphi}\right)
\end{aligned}
$$

Now it can finally be rewritten as

$$
\begin{aligned}
& \sigma_{11}\left(\frac{d S_{1111}}{d \varphi}-4 S_{1112}\right)+\sigma_{22}\left(\frac{d S_{1122}}{d \varphi}+2 S_{1112}-2 S_{1222}\right)+ \\
& +2 \sigma_{12}\left(\frac{d S_{1112}}{d \varphi}+S_{1111}-S_{1122}-2 S_{1212}\right)=0
\end{aligned}
$$

Again, this holds for the arbitrary stress tensor and therefore the expressions in all the parentheses of (25) must vanish. Considering (20), it means that

$$
\frac{d S_{1111}}{d \varphi}=4 S_{1112} \quad, \quad \frac{d\left(\frac{1}{E_{x}}\right)}{d \varphi}=2 \alpha_{x} \quad Q D E
$$

So this proves that (8) holds generally, and not only in the case of an orthotropic material. Further relations between elastic material properties and their derivatives according to the second and the third parenthesis in (25) would read

$$
\frac{d}{d \varphi}\left(\frac{v_{y x}}{E_{x}}\right)=\alpha_{x}-\alpha_{y} \quad, \quad \frac{d \alpha_{x}}{d \varphi}=\frac{1}{G_{x y}}-\frac{2}{E_{x}}-2 \frac{v_{y x}}{E_{x}}
$$

It is left here for interested readers to verify that expressions (6) do satisfy equations (27). A similar treatment of other component equations of (19) would obviously lead to more relations between elastic material properties in the rotation of the coordinate system - this stretches beyond the scope of the present analysis and is also left to the readers. Maybe some of the relations obtained in that way would coincide with the already developed (26) or (27).

\section{Examples}

In the examples presented here some characteristic theoretical models suitable for the description of elasticity of textile materials are considered: woven fabric in a basic weave pattern (plain weave) with full structural symmetry, a flat stripe composite, and a combination thereof which can be regarded as an approximate model of woven fabric with a diagonally structured weave pattern (twill weave or the like). The fact is that the tensile response of woven or knitted fabric is nonlinear, since the structural geometry changes with deformation, and the combination of nonlinearity and anisotropy may become demanding. Still, it does make sense to apply the methodology of polar diagrams of elastic material properties, at least in a limited proximity of a given starting point where the elastic response can be linearized. 


\subsection{Fabric woven in plain weave}

This is the simplest weave pattern where warp and weft yarns are completely interlaced, without any skipping. Since the tension in the warp direction is higher during the weaving process, it is usual that the tensile modulus is higher in the warp direction and lower in the weft direction. Full structural symmetry with respect to both the warp and the weft direction results also in deformational symmetry, so here we deal with an orthotropic material. In Fig. 4 polar diagrams of the tensile modulus and the normal-shear coefficient are shown for the case $\mathrm{E}_{1}=1, \mathrm{E}_{2}=1.5, v_{12}=0.25, \mathrm{G}_{12}=0.1$.

a)

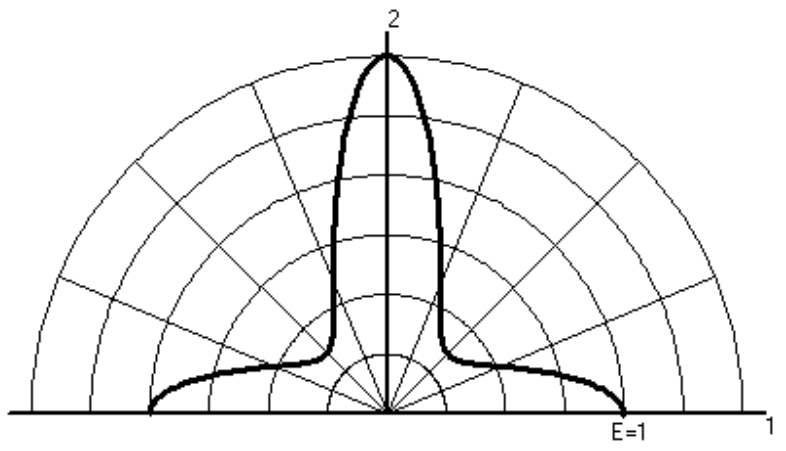

b)

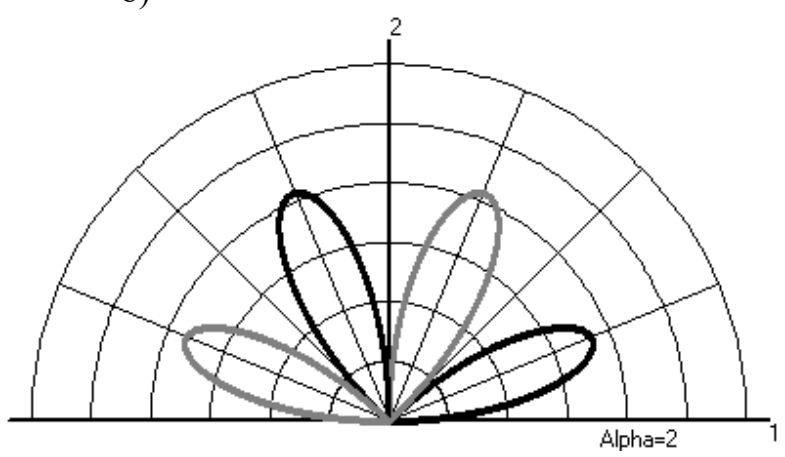

Fig. 4 Example 1, polar diagrams of tensile modulus (a), and of normal-shear coefficient (b)

Weft and warp directions are denoted by 1 and 2, respectively, and here they coincide with the principal material directions. The moduli are normalised with respect to the weft tensile modulus. In the case of a negative normal-shear coefficient, absolute value is presented in grey. Note how the normal-shear coefficient can be related to the rate of change of the tensile modulus in accordance with (8). A sharp drop of the tensile modulus in the diagonal direction with respect to warp and weft ("bias direction") is associated with the low value of the shear modulus, particularly pronounced in fabrics with a low yarn density. As far as the units are concerned, if, for example, the tensile moduli were in $\mathrm{GPa}$, it would mean that the normal-shear coefficients are in $\mathrm{rad} / \mathrm{GPa}$.

\subsection{Flat stripe composite}

Here we consider a theoretical model of a material consisting of stripes made from two different simple (isotropic linear elastic) materials. Imagine, for example, that in the fabric for a marine T-shirt white and dark stripes have different elastic properties.

Theoretical homogenisation of elastic properties of such a material, based on static and compatibility conditions of the basic cell, has been reported in [1] and [5] and, basically, it reduces to the principal elastic moduli obtained as the arithmetic mean value (along the stripes) and the harmonic mean value (perpendicular to stripes) of individual material elastic moduli - this is similar to equivalent stiffness of springs in a parallel and serial assembly with certain correction due to differences in the elastic moduli and non-zero Poisson's ratios. As for the shear modulus of such a composite, it may be concluded that it is not totally unique and can range from the harmonic to the arithmetic mean value of individual material shear moduli, depending on whether boundary restrictions are expressed in terms of loads or displacements.

In the present example the following case is considered: individual elastic moduli of ingredient materials are different by factor $5, \mathrm{E}_{\mathrm{A}}=5 \mathrm{E}_{\mathrm{B}}$, Poisson's ratios are equal, $v_{\mathrm{A}}=\mathrm{v}_{\mathrm{B}}=0.25$. The homogenisation leads to principal elastic moduli which, when expressed in a relative 
Normal-Shear Coefficients and

Tensile Moduli in Anisotropic Materials

way, i.e. normalised with respect to $E_{1}$, have the values $E_{1}=1$ and $E_{2}=0.5714$. Shear modulus can then be in the range from 0.2222 to 0.4 .

Polar diagrams of the elastic modulus and the normal-shear coefficient for the case of $\mathrm{G}_{12}=0.3$ are shown in Fig. 5.

Let us note that the polar diagrams of the elastic moduli in the cases of extreme possible shear moduli have a less natural shape than the elliptic shape presented in Fig. 5, as indicated in Fig. 6.

a)

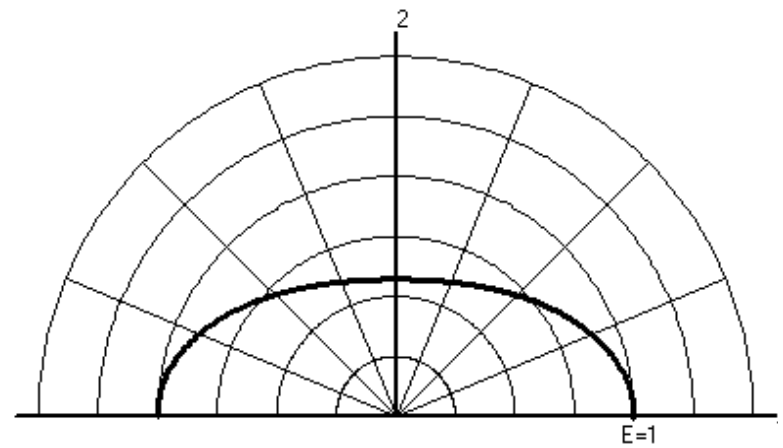

b)

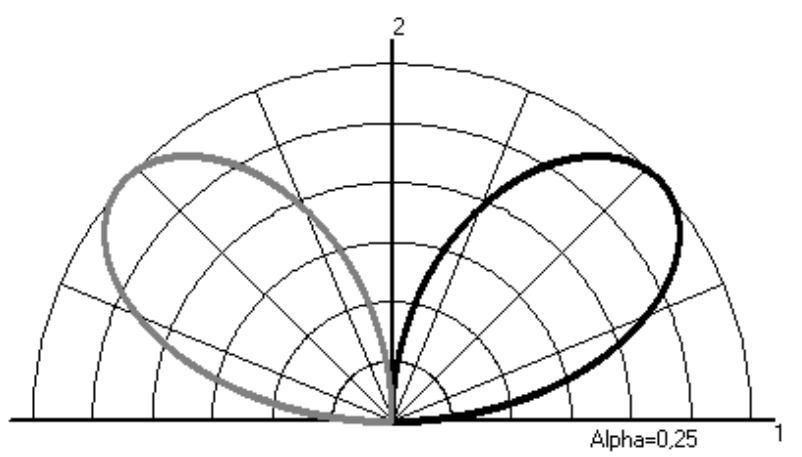

Fig. 5 Example 2, polar diagrams of tensile modulus (a), and of normal-shear coefficient (b), case $\mathrm{E}_{1}=1$, $\mathrm{E}_{2}=0.5714, v_{12}=0.25, \mathrm{G}_{12}=0.3$

a)

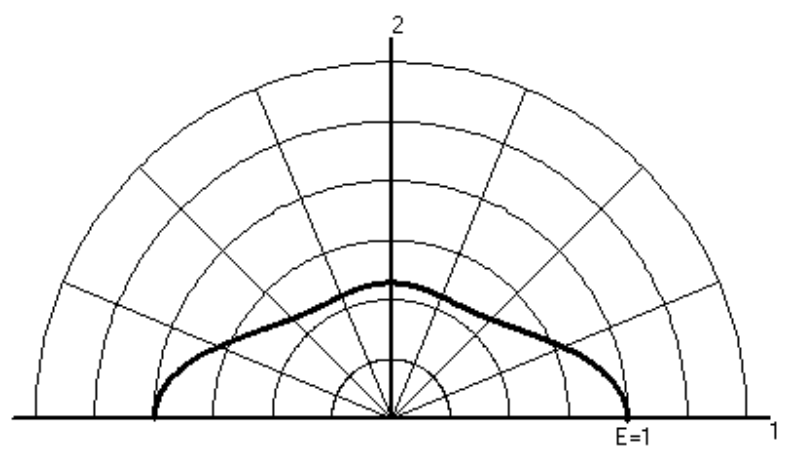

b)

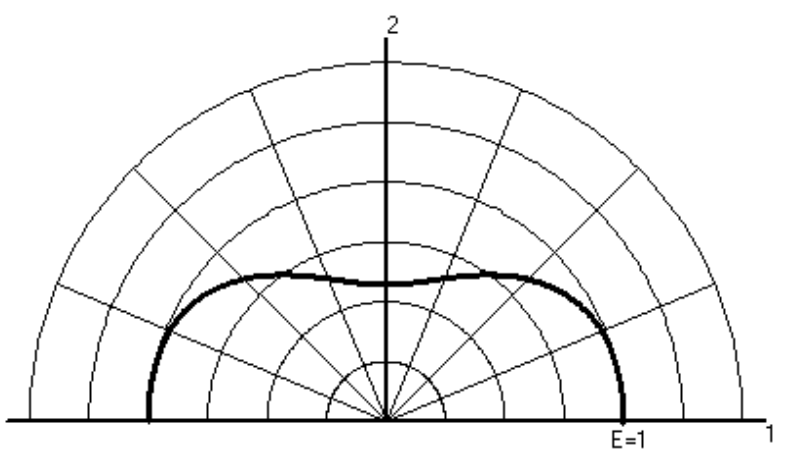

Fig. 6 Example 2, polar diagrams of tensile modulus with $\mathrm{G}_{12}=0.2222$ (a), and with $\mathrm{G}_{12}=0.4$ (b)

\subsection{Woven fabric with diagonal weave pattern}

In the cases of weave patterns where yarns are skipped in places rather than fully interlaced, structural symmetry may be lost. This is the case, for example, with twill weave, Fig. 7a. Warp and weft yarns are depicted in different colours and it can be seen that the areas of different structures are distributed diagonally with respect to the yarns, thus the term diagonal pattern. A simplified approximate theoretical model of deformational properties of such a fabric may be constructed taking into account two principal influences: warp and weft yarns, e.g. in a symmetric pattern, as primary influence, and diagonal distribution of areas with different structures as secondary influence. Therefore it is proposed here to construct a model for such a fabric combining the cases of examples 1 and 2 to obtain a theoretical composite with different weight factors and stripes spaced diagonally to warp and weft. A polar diagram of the elastic modulus is shown in Fig. $7 \mathrm{~b}$ for such a model for the cases of examples 1 and 2 with weight factors 0.6 and 0.4 , respectively. It is observed that there is no elastic symmetry, so this is no longer an orthotropic but rather a general anisotropic material and axes 1 and 2 are no longer axes of extreme elastic moduli. In other words, there are non- 
zero normal-shear coefficients in the directions of warp and weft, a deformational property of some woven fabric which is sometimes confusing and causes problems in fabric processing.

a)

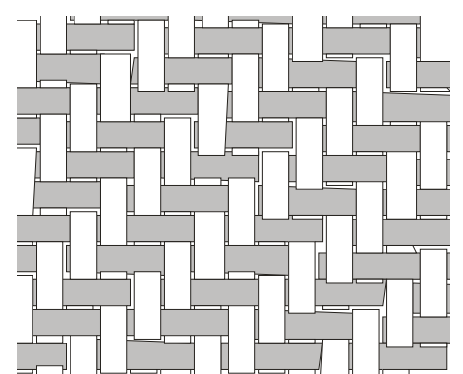

b)

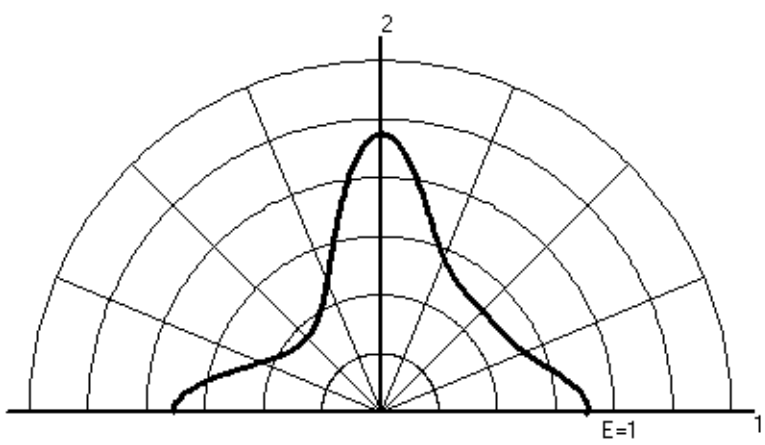

Fig. 7 Example 3, fabric in twill weave (a), and polar diagram of elastic modulus for combination of examples 1 and 2 with factors 0.6 and 0.4 , stripes diagonal to yarns (b)

\section{Conclusions}

The relation between the normal-shear coefficient and the elastic modulus (8) observed in the transformation formulae for the elastic compliance of an orthotropic material has been derived generally for the case of an anisotropic material. It appears that there are a number of similar relations among elastic material properties in the rotation of the coordinate system, which can be derived from the transformation rules of the second order tensors of stress and strain. The polar diagram methodology appears to be suitable for the representation of elastic properties of anisotropic materials, notably the relation between elastic moduli and normalshear coefficients has a clear geometric representation. Some theoretical examples are presented that can be related to woven fabrics and textile composites, including nonsymmetric properties of the fabric with a diagonal weave pattern.

\section{REFERENCES}

[1] Šomođi, Ž.; Kovačević, S.; Dimitrovski, K.: Weft Distortion after Weaving - An Approximate Theoretical Model, Proceedings of $5^{\text {th }}$ International Textile, Clothing \&Design Conference, Dragčević, Z., Hursa Šajatović, A. \& Vujasinović, E. (Eds.), pp. 729-734, ISSN 1847-7275, Dubrovnik, October 2010, Faculty of Textile Technology, University of Zagreb, Zagreb, Croatia, (2010)

[2] Alfirević, I.:Linear Structural Analysis:Thin-walled Structures, Protei, Zagreb, 2005.

[3] Alfirević, I.: Uvod u tenzore i mehaniku kontinuuma (Introduction to tensors and continuum mechanics, in Croatian), Golden marketing, Zagreb, 2003.

[4] Alfirević, I.; Skozrit, I: Linear, quadratic and cubic invariants of orthotropic elastic materials, Transactions of FAMENA, 30 (2006), 1; 17-26

[5] Šomođi, Ž.; Brnada, S.; Kovačević, S.: Elements of Anisotropy in Woven Fabrics and Composites, Proceedings of $8^{\text {th }}$ International Textile, Clothing \&Design Conference, Dragčević, Z., Hursa Šajatović, A. \& Vujasinović, E. (Eds.), pp. 729-734, ISSN 1847-7275, Dubrovnik, October 2016, Faculty of Textile Technology, University of Zagreb, Zagreb, Croatia, (2016)

Submitted: $\quad 06.7 .2017$

Accepted: $\quad 18.10 .2017$
Željko Šomođi (corresponding author)

Emilija Zdraveva University of Zagreb, Faculty of Textile Technology, Prilaz B. Filipovića 28a zeljko.somodi@ttf.hr 